\title{
(2) OPEN ACCESS \\ Spectral domain optical coherence tomography-based retinochoroidal cystine crystal score: a window into infantile nephropathic cystinosis
}

\author{
Leonie Keidel 다, ' Katharina Hohenfellner, ${ }^{2}$ Benedikt Schworm, ${ }^{1}$ Siegfried Priglinger, \\ Nikolaus Luft, ${ }^{1}$ Claudia Priglinger ${ }^{1}$
}

1 Department of Ophthalmology, University Hospital, LMU Munich, Munich, Bayern, Germany

${ }^{2}$ Department of Pediatric Nephrology, RoMed Kliniken Rosenheim, Bayern, Germany

Correspondence to Dr Claudia Priglinger, Ophthalmology, Ludwig-

Maximilians-Universitat Munchen, Bayern, 80539 München, Germany; claudia.priglinger@med.unimuenchen.de

$\mathrm{NL}$ and CP contributed equally.

Received 6 May 2021

Accepted 17 August 2021

Check for updates

(C) Author(s) (or their employer(s)) 2021. Re-use permitted under CC BY-NC. No commercial re-use. See rights and permissions. Published by BMJ.

\section{To cite: Keidel $\mathrm{L}$} Hohenfellner K, Schworm B, et al. Br J Ophthalmol Epub ahead of print: [please include Day Month Year]. doi:10.1136/

bjophthalmol-2021-319612

\section{ABSTRACT}

Précis Cystinosis is a lysosomal storage disease leading to an accumulation of cystine crystals in several organs. We aim to comprehensively describe chorioretinal cystine crystals via spectral domain optical coherence tomography (SD-OCT) and elaborate a new biomarker for systemic disease control.

Background/aims Cystinosis is a rare lysosomal storage disease leading to an accumulation of cystine crystals in several organs. This study aims to describe the deposition of retinochoroidal crystals in infantile nephropathic cystinosis and to elucidate their potential value as an objective biomarker for systemic disease control.

Methods This cross-sectional study was carried out by the University Eye Hospital of the LudwigMaximilian University (Munich, Germany) in collaboration with the German Cystinosis Study Group. A complete ophthalmologic examination was performed, along with posterior segment SD-OCT (Spectralis; Heidelberg Engineering $\mathrm{GmbH}$, Heidelberg, Germany). Retinochoroidal crystals were graded by employing a novel semiquantitative grading systemthe retinochoroidal cystine crystal score (RCCCS). To quantify quality of vision, patients completed a specific questionnaire. A total of 85 eyes of 43 patients with cystinosis were included (mean age $22.3 \pm 8.8$ years, range 6-39; male:female ratio=23:20).

Results Cystine crystals were detectable in all neuroretinal layers and the choroid, most frequently in the choriocapillaris. The RCCCS was negatively correlated with cysteamine intake $(r=0.533, p=0.001)$ and positively with cystatin $C$, a stable parameter of renal function ( $r=0.496, p=0.016)$. Moreover, the value of the RCCCS affected subjective quality of vision. Genetic analysis indicated pronounced crystal deposition in patients with heterozygous mutations containing the 57-kb-deletion allele of the CTNS gene.

Conclusion Ocular cystinosis leads to retinochoroidal crystal accumulation in every stage of the disease. Crystal deposition may be markedly influenced by oral cysteamine therapy. Therefore, the presented SD-OCT based grading system might serve as an objective biomarker for systemic disease control.

\section{INTRODUCTION}

Infantile nephropathic cystinosis is a rare lysosomal storage disease that causes an accumulation of cystine crystals in every tissue of the body including various ophthalmic structures. ${ }^{1}$ Due to a defective cystine transporter in the lysosomal membrane, intracellular cystine precipitates and leads to malfunction of several organs. ${ }^{2}$ Renal Fanconi syndrome, for instance, is most often caused by cystinosis in children. Cystinosis also leads to end stage renal failure requiring dialysis or renal replacement therapy. ${ }^{34}$ Regarding the ocular manifestations of infantile nephropathic cystinosis, cystine crystals can be found in the cornea, conjunctiva, iris, ciliary body, choroid and retina. ${ }^{5} 6$

Oral administration of the free thiol cysteamine is the current treatment of choice for the systemic manifestations of cystinosis. ${ }^{7}$ Cysteamine depletes cystinotic cells by up to $90 \%$ of their cystine content. $^{8}$ When administered early and regularly, it may protract systemic manifestations (eg, nephropathy or retinopathy) and increase overall life expectancy. ${ }^{9}$ Yet, due to the oral therapy's various side effects, it is often difficult to achieve therapeutic compliance, especially in paediatric and adolescent individuals. ${ }^{10}$ Patients often suffer from nausea, vomiting and sulfurous body and breath odour, which may cause psychological and social problems. $^{11}$

To date, the white blood cell (WBC) cystine level obtained through blood sampling is the only existing and relevant biomarker used to monitor the success of oral cystine-depleting therapy. This biomarker, however, does not reflect long-term compliance with cysteamine treatment. WBC cystine levels vary considerably, largely depending on the period since the last oral intake of cysteamine. ${ }^{12}$ Hence, a more representative and stable biomarker is warranted to efficiently monitor the success of, and long-term adherence to, systemic therapy; such monitoring might help foster patient compliance.

The eye with its physiologically clear optical media-previously described as 'the window to cystinosis ${ }^{5}$ - is ideally accessible to analysis with optical imaging. Since the retinal and choroidal circulation are connected to the systemic blood flow, retinochoroidal cystine crystals may be affected by oral cystine-depleting therapy. ${ }^{13}$ Spectral domain optical coherence tomography (SD-OCT) is a non-invasive imaging modality that enables cross-sectional scans of retinal and choroidal tissues in a close histological resolution of up to $1-4 \mu \mathrm{m}$ axial resolution; it can be considered an in vivo biopsy'. ${ }^{14}$ Patients with cystinosis suffer from severe photophobia-presumably due to cystine crystals in the cornea, which cause incoming light to scatter. ${ }^{15}$ 
Therefore, SD-OCT is a particularly good diagnostic tool for cystinosis individuals because it uses light in the near-infrared spectral range, which is invisible for the human eye and therefore cannot cause photophobia. ${ }^{16}$

This study aims to comprehensively describe and characterise the deposition of retinochoroidal crystals through SD-OCT in a large cohort of patients with cystinosis. Another goal was to gauge their potential value as an objective biomarker for systemic disease control.

\section{MATERIALS AND METHODS}

This cross-sectional study recruited patients with infantile nephropathic cystinosis from the German Interdisciplinary Cystinosis Clinic Germany between 2018 and 2019. ${ }^{17}$ Consent to use their data for analysis and scientific publication was obtained from all participating patients. All ophthalmological examinations were carried out at the University Eye Hospital, Ludwig-Maximilian University, Munich, Germany.

\section{Ophthalmological examination}

A complete ophthalmological examination was performed consisting of slit lamp biomicroscopy, dilated funduscopy, Goldmann applanation tonometry and best corrected visual acuity (BCVA) using the standard ETDRS chart for testing at a distance of $4 \mathrm{~m}$.

\section{Spectral domain optical coherence tomography}

An SD-OCT system (Spectralis; Heidelberg Engineering GmbH, Heidelberg, Germany) was used for B-scan acquisition. In all cases, macular volume scans consisting of 25 B-scans $(512$ A-scans each), covering a $20^{\circ} \times 20^{\circ}$ region centred at the fovea, were obtained. Image optimisation using the automatic realtime (ART) averaging algorithm (12 frames per B-scan) of the onboard manufacturer's software was employed. The singular horizontal B-scan with the best centration in relation to the foveolar specular reflex was used for further image analysis. In cases where the acquisition of a volume scan was not possible due to non-compliance (5 eyes of 3 patients), a singular line scan centred on the foveola with ART averaging (2 frames per B-scan) was taken and used for analysis instead.

As previously described, ${ }^{16}$ retinal crystals appear as distinct hyperreflective foci on SD-OCT B-scan images. For the purpose of this study, hyperreflective foci representing cystine crystals were graded by two independent expert SD-OCT readers (LK, $\mathrm{CP}$ ), who did not know the patients' identities or details about their ocular or systemic health. One singular central horizontal B-scan image was analysed per eye. Crystals were semiquantitatively analysed for the entire view field of the B-scan image, separately for each of the following retinochoroidal layers: retinal nerve fibre layer (RNFL), ganglion cell layer (GCL), inner plexiform layer (IPL), inner nuclear layer (INL), outer plexiform layer (OPL), outer nuclear layer (ONL), and choroid. The retinal pigment epithelial (RPE) and photoreceptor layer were not included in the present analysis, because the difference in pixel brightness between cystine foci and physiological tissue of the concerned layers was too similar to be differentiated with certainty. The retinochoroidal cystine crystal score (RCCCS) grading system is constituted by four semiquantitative values: $0.5+: 1-3$ crystals present in the concerned retinochoroidal layer, 1+: 4-10 crystals, 2+: 11-20 crystals, 3+:>20 crystals. By adding up all the semiquantitative values for the seven individual retinochoroidal layers, the RCCCS (representing cumulative crystal deposition in all retinochoroidal layers) was calculated for each eye. The RCCCS can range from 0 (indicating no deposition) to 21 (indicating maximum, ie $>20$, crystal deposition in all retinochoroidal layers). In cases where the grading between the two independent SD-OCT readers were not congruent (36 of 595 individual retinochoroidal layer gradings; $6.1 \%$ ), a consensus grading was performed.

\section{Measuring corneal cystine crystal deposition}

Corneal crystals were graded using the objective grey valuebased parameter developed by our group and according to the already established subjective Gahl-Corneal Cystine Crystal Score (CCCS). ${ }^{5}$ For objective grading of the corneal crystals, a semiautomated grey scale system was developed using anterior segment optical coherence tomography (Cirrus HD-OCT 5000, Carl Zeiss Meditec AG, Jena, Germany) and applied as described previously. ${ }^{6}$ The CCCS, on the other hand, is a subjective 13-step grading system based on a library of slit-lamp photographs of corneas with increasing crystal density. The assessment is made by subjective image comparison with an online photo atlas.

\section{Patient-reported outcome measures}

To quantify patient-reported, subjective quality of vision, patients were asked to complete a standardised, Rasch-scaled, clinically validated 30-item questionnaire addressing different subjective visual symptoms. ${ }^{18}$ The questionnaire was designed to quantify the frequency, severity and irritation level of the following visual symptoms: glare perception, halo perception, starbursts, foggy vision, blurred vision, distorted vision, double vision, fluctuations in vision, focusing difficulties, and difficulties judging distance or depth perception. All but the latter three symptoms were illustrated by standardised colour images printed in standardised size $(163 \times 232 \mathrm{~mm}, 300 \times 300 \mathrm{DPI})$. Patients were encouraged to report how often (never (0), occasionally (1), quite often (2), very often ${ }^{19}$ ) and how severe (not at all (0), mild (1), moderate (2), severe ${ }^{19}$ ) they experienced each symptoms and how much they were bothered by them (not at all $(0)$, a little (1), quite (2), very $^{19}$ ).

\section{Blood sampling and genetic testing}

Blood was drawn for genetic testing and determination of the WBC cystine level and cystatin C levels (Institute for Laboratory Medicine, Ludwig-Maximilian University, Munich). To analyse the influence of renal function on RCCCS, only the cystatin C levels of patients who had not yet undergone kidney transplantation were included in the calculation.

To evaluate the impact of different allele combinations on chorioretinal crystal distribution, the percentage of decisions, in which an accumulation of crystals of stage $0.5,1,2,3$ according to the RCCCS grading system was described by the graders, was divided by all the gradings that had to be made in this specific group with the same mutation.

\section{Statistical analysis}

All statistical analyses were performed using SPSS Statistics (V.25.0; IBM). All metric data were presented as mean $\pm S D$; ordinal data were presented as median and range. For the RCCCS, we used the mean value of the two eyes for all calculations. Correlation analyses between the RCCCS and age, as well as BCVA, the WBC cystine level and cystatin $\mathrm{C}$ levels and the dose of oral cysteamine intake were performed by calculating Pearson's correlation coefficient. Spearman correlation analyses were employed to assess the associations between retinochoroidal 
Table 1 Subjects' examination results and daily dose of the systemic medication

\begin{tabular}{lll}
\hline Parameter & Mean \pm SD & Range \\
\hline $\begin{array}{l}\text { Best corrected visual acuity } \\
\text { (logMAR) }\end{array}$ & $0.03 \pm 0.17$ & $-0.21-1.00$ \\
$\begin{array}{l}\text { Daily dose of cysteamine } \\
\text { (mg/kg body weight/day) }\end{array}$ & $37.61 \pm 16.31$ & $0.00-66.70$ \\
$\begin{array}{l}\text { Serum cystatin C level (mg/L) } \\
\text { (Normal range: } 0.6-1 \mathrm{mg} / \mathrm{L} \text { ) }\end{array}$ & $1.94 \pm 1.31$ & $0.69-7.08$ \\
$\begin{array}{l}\text { WBC cystine }(\mathrm{nmol} / \mathrm{mg} \text { protein) } \\
\text { (normal range: }<1 \mathrm{nmol} / \mathrm{mg} \text { protein) }\end{array}$ & $1.02 \pm 1.11$ & $0.07-5.70$ \\
\hline
\end{tabular}

WBC, white blood cell.

crystal accumulation and subjective visual symptoms. A p value of $<0.05$ was considered to indicate statistical significance.

\section{RESULTS}

This study included a total of 85 eyes belonging to 43 patients with infantile nephropathic cystinosis. The female to male ratio was 20:23 and mean age was $22.3 \pm 8.8$ (range 6-39) years. Further characteristics of the examined cohort are summarised in table 1.

Retinochoroidal cystine crystals are hardly visible in funduscopy and therefore cannot be precisely quantified without a sectional image. For this reason, OCT was used to develop the RCCCS grading system (figure 1A-C). Retinochoroidal cystine crystals as indicated by hyperreflective foci in the SD-OCT B-scan image were encountered in 58 of 85 eyes $(68.2 \%$; figure 2). Crystal deposition was detected in all neuroretinal layers as well as in the choroid. Crystals in the choroid were the most common SD-OCT manifestation affecting 53 eyes (62.4\%, figure 3A). Choroidal crystals were confined to the choriocapillaris layer in all cases (figure $2 \mathrm{~A}$ and $\mathrm{B}$ ). The second and third most common layers affected by crystal deposition were the INL with 18 (21.2\%) and GCL with 14 (16.5\%) eyes (figure 3A) which showed hyperreflective foci on SD-OCT. Crystals of the INL were predominantly found in proximity to
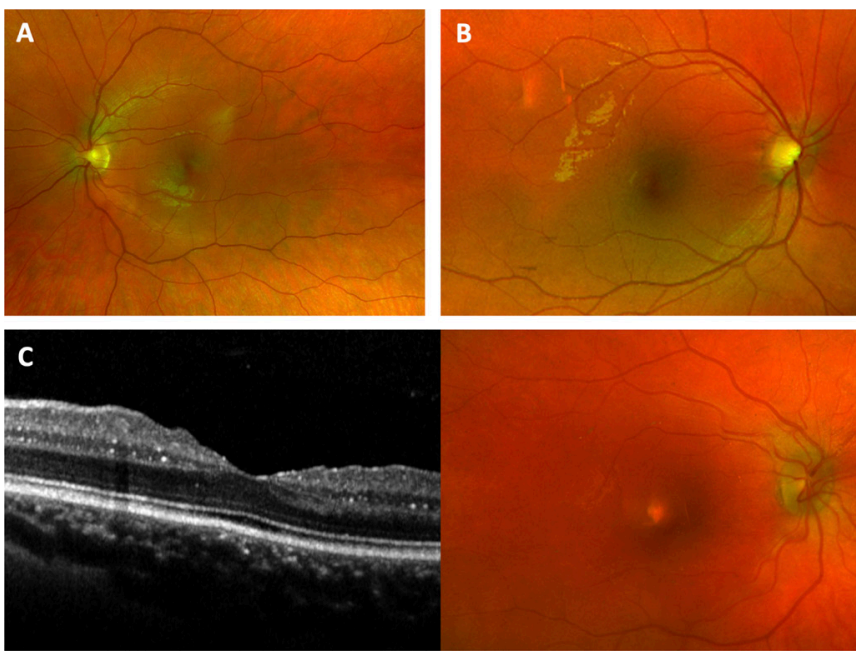

Figure 1 Fundus photographies of patients with cystinosis. Matched fundus photography of the patient with cystinosis shown in figure $2 \mathrm{~A}$ with a retinochoroidal cystine crystal score (RCCCS) of (A) 0.5 and (B) 2.5, respectively. (C) Optical coherence tomography and fundus photography of a patient with an RCCCS of 2.25. Chorioretinal crystals are hardly visible on funduscopy and fundus photographies.
A

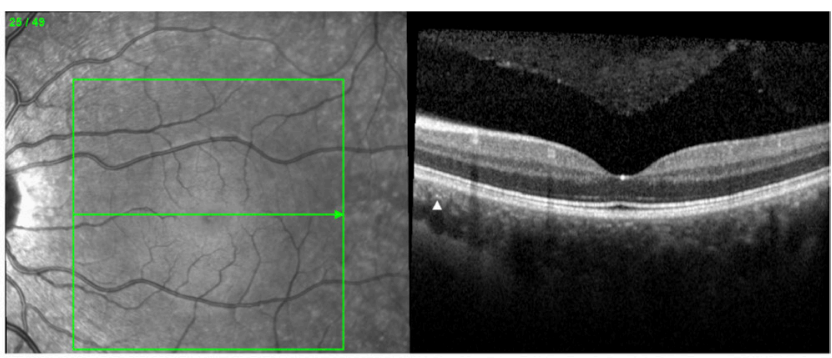

B

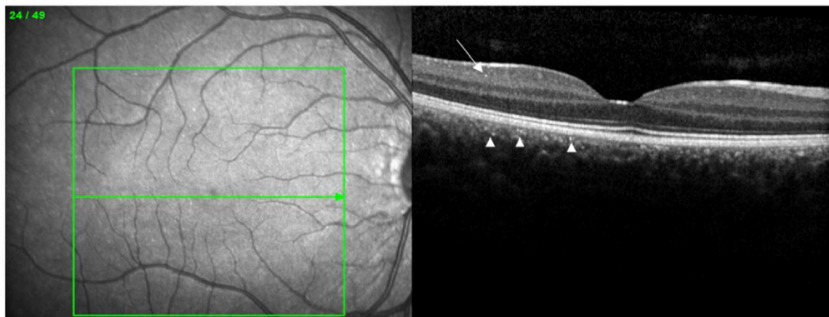

C

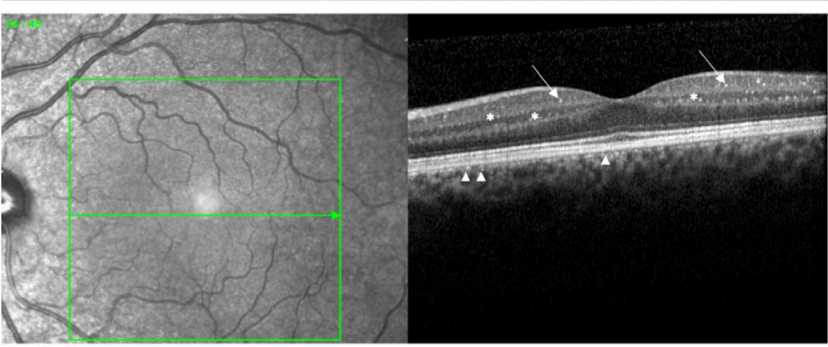

D

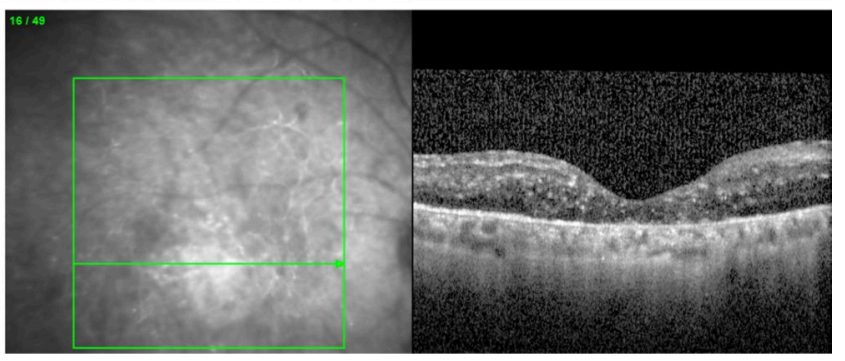

Figure 2 Spectral domain optical coherence tomography B-scans of patients with cystinosis. (A) Retinal crystals presenting as hyperreflective foci with a pronounced accumulation in the choriocapillaris (arrowhead). Retinochoroidal cystine crystal score (RCCCS) of 0.5 with only few choroidal crystals. (B) RCCCS of 2.5 with more crystals in the choroid (arrowhead) and inner plexiform layer (IPL). ${ }^{36}$ (C) RCCCS of 5 with crystals in the choroid, GCL, IPL and inner nuclear layer (arrows). Crystals were most often found at the transition zones between the layers. (D) Severe crystal accumulation in multiple chorioretinal layers.

the transition zones towards the adjacent OPL and IPL, respectively (figure $2 \mathrm{C})$. Crystal deposition in the IPL $(\mathrm{n}=9 ; 10.6 \%)$, RNFL $(n=5 ; 5.9 \%)$, OPL $(n=3 ; 3.5 \%)$ and ONL $(n=1 ; 1.2 \%)$ were less common. In some cases, severe crystal deposition lead to retinal atrophy (figure 2D).

\section{Genotype and retinochoroidal crystal deposition}

The molecular genetic testing results are displayed in figure 3B. Low grade of crystal deposition was quite evenly distributed over most mutations and the heterozygous combinations thereof. Overall, crystal deposition was most common in patients with heterozygous mutations containing the $57-\mathrm{kb}$-deletion allele of the CTNS gene combined with c.18_21delGACT or the amino acid substitution at c. $1118 \mathrm{~T}>\mathrm{C}$, respectively. Most pronounced retinal crystal deposition was found in patients heterozygous for 
A

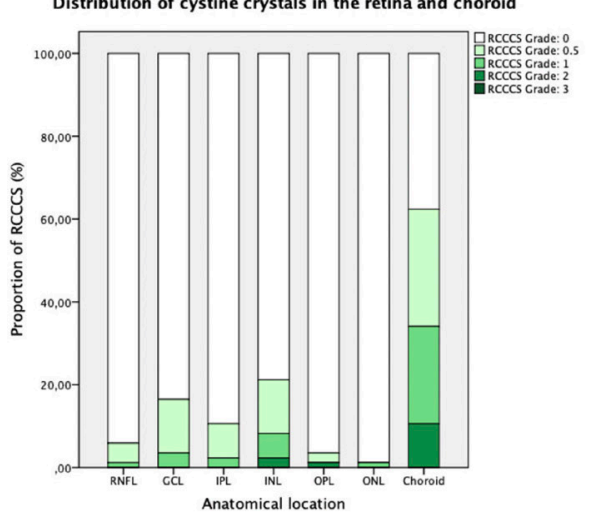

B

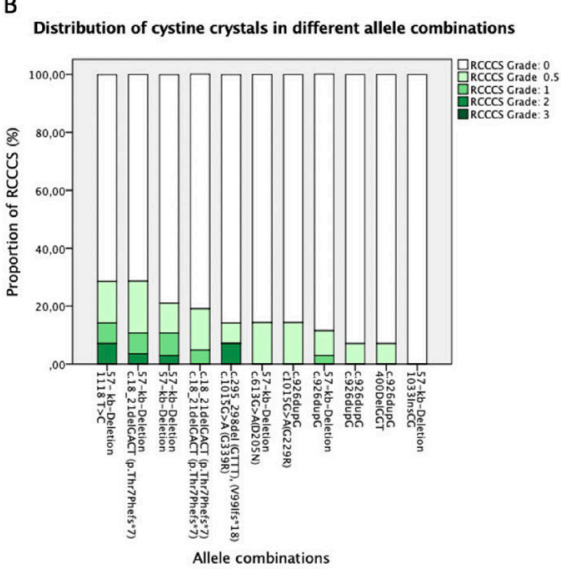

Figure 3 Display of the cystine crystal distribution in the retina and choroid and in different allele combinations. (A) Distribution of cystine crystals with respect to their localisation in different retinal layers and the choroid and (B) with respect to their occurrence in different genetic mutations.

c.295_298del and c.1015G $>$ A as well as the combination of the 57-kb-deletion with c. $1118 \mathrm{~T}>\mathrm{C}$ (figure $3 \mathrm{~B}$ ).

\section{Systemic determinants of retinochoroidal crystal deposition}

A propensity towards higher RCCCS values with increasing patient age at the time of examination was detected $(r=0.294$, $\mathrm{p}=0.056$ ). The RCCCS was found as a statistically significant positive function of serum cystatin $C$ concentration $(r=0.496$, $\mathrm{p}=0.016$; figure 4A). Moreover, RCCCS negatively correlated with the daily dose of cysteamine in relation to body weight

A

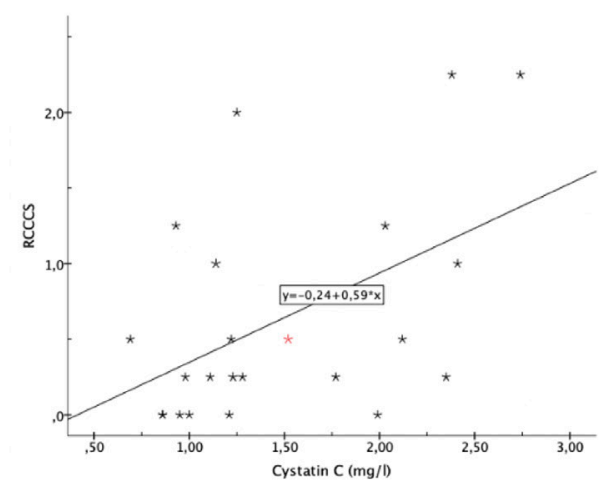

C

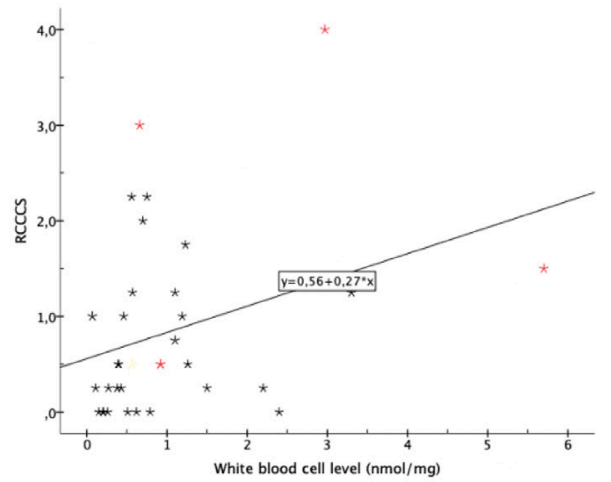

$(\mathrm{r}=0.533, \mathrm{p}=0.001$; figure $4 \mathrm{~B})$ and showed a propensity towards higher values with increasing WBC cystine level $(r=0.327$, $\mathrm{p}=0.055$; figure $4 \mathrm{C}$ ).

\section{Retinochoroidal crystal deposition and visual function}

Higher RCCCS values were statistically significantly associated with reduced BCVA $(r=0.465, p<0.01$, figure 4D). Regarding questionnaire-reported visual symptom outcomes, glare was the most commonly perceived visual symptom with a cumulative $84.8 \%$ of eyes reporting glare perception 'occasionally' (48.1

\section{B}

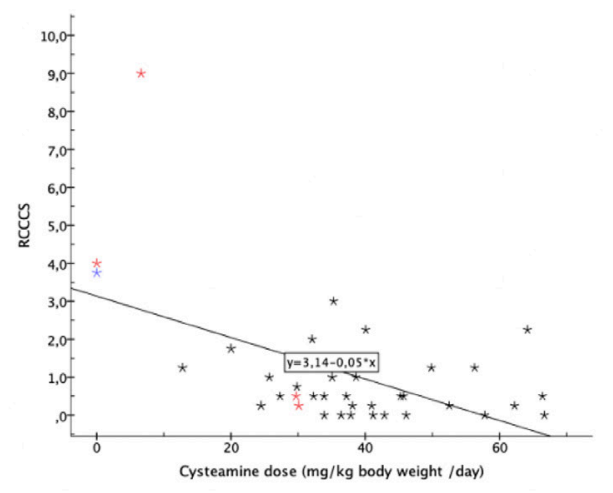

D

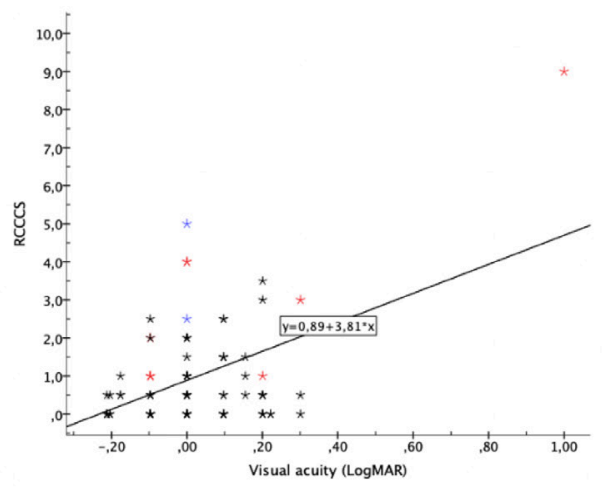

Figure 4 Correlation of the retinochoroidal cystine crystal score (RCCCS) with systemic parameters, daily dose of cysteamine and visual acuity. (A) Positive correlation with the cystatin C level in the blood in pretransplant patients with cystinosis. (B) Inverse correlation with the dose of cysteamine intake. (C) Patients showing a higher white blood cell cystine level presented with a higher RCCCS. (D) Inverse correlation with best corrected visual acuity. 
A

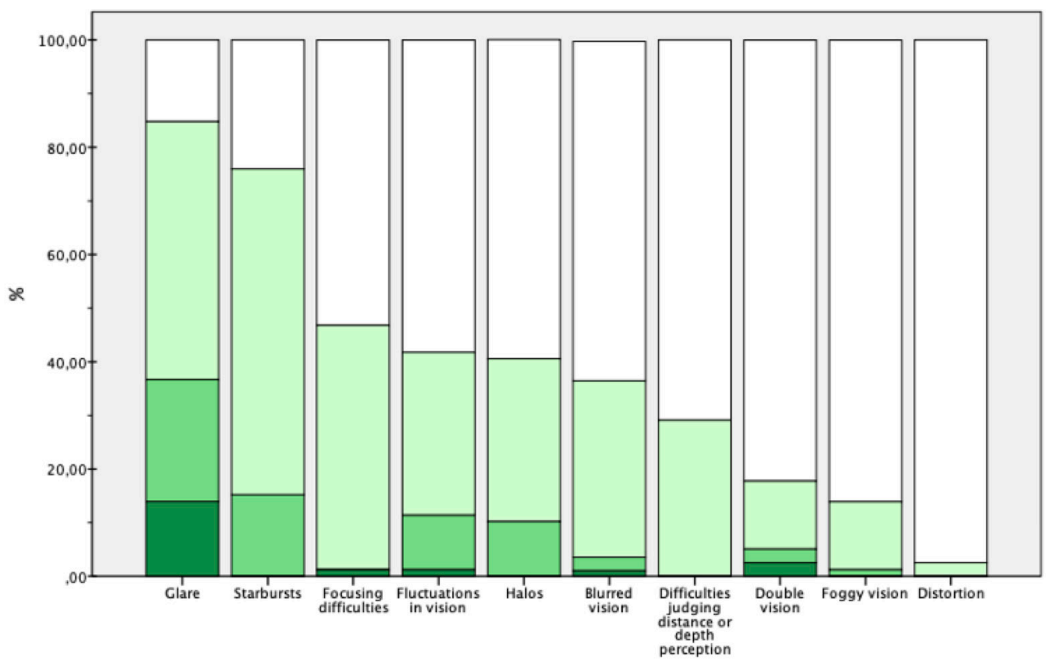

B

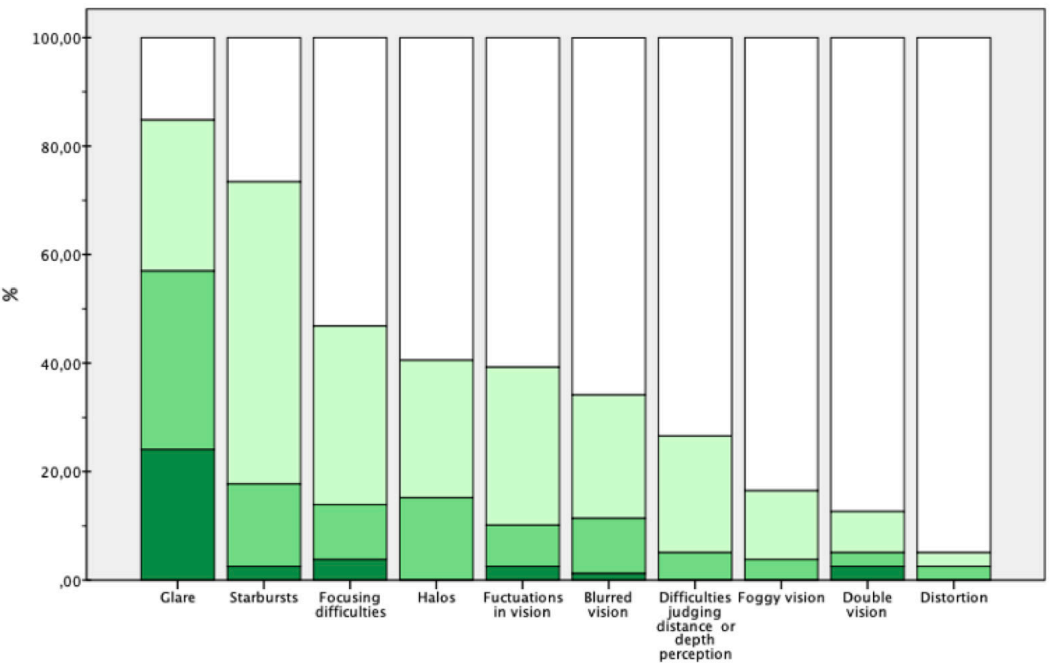

C

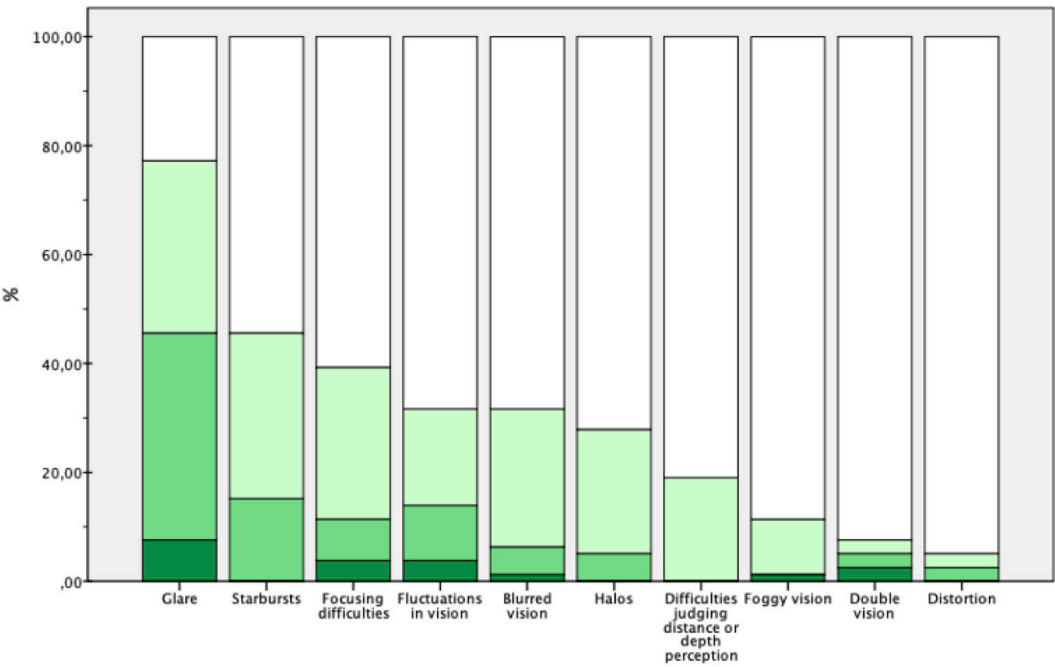

Frequency

品ever

Guccasionally

Quite often
Very often

Figure 5 Impact on subjective quality of vision. (A) Frequency, (B) severity and (C) bothersome of the perception of different quality of vision parameters. Patients suffered in descending frequency mostly from the perception of glare, starbursts and focusing difficulties.

$\%$ ), ‘quite often' $(22.8 \%)$ or 'very often' (13.9\%; figure 5). Glare was both the most severely perceived and most irritating of all tested visual symptoms. Starburst perception was the second most commonly reported (75.9\%) symptom and was also severely perceived and irritating (figure 5). The remaining eight visual symptoms (eg, foggy vision) were relatively less reported 
with cumulative incidences ranging between $46.8 \%$ for focusing difficulties and $2.53 \%$ for image distortion.

While the frequency $(\mathrm{Rho}=0.182, \mathrm{p}=0.262)$ of glare perception was not a function of RCCCS, severity and irritation levels of glare showed a statistically significant positive association with RCCCS $(\mathrm{Rho}=0.432, \mathrm{p}=0.05$ and $\mathrm{Rho}=0.313, \mathrm{p}=0.049)$. Neither the frequency $(R h o=0.131, p=0.420)$ nor the severity $(\mathrm{Rho}=0.157, \mathrm{p}=0.334)$ or the irritation level $(\mathrm{Rho}=0.140$, $\mathrm{p}=0.388$ ) of starburst perception was associated with RCCCS.

\section{Retinochoroidal crystal deposition and corneal crystal accumulation}

There was no correlation between the occurrence of chorioretinal crystals and the presence of corneal cystine crystals measured by the CCCS $(R=0.199 p=0.206)$ or via the OCT-based grey scale grading system $(\mathrm{R}=0.270, \mathrm{p}=0.106)$.

\section{DISCUSSION}

The present study shows that infantile nephropathic cystinosis leads to retinochoroidal crystal accumulation and that there is a predominant deposition in the choriocapillaris, as well as in the INL and GCL. This is the first study to provide evidence that retinochoroidal crystal accumulation can be positively influenced by systemic cysteamine therapy, although its occurrence cannot be completely be prevented.

Our SD-OCT imaging-based in vivo findings corroborate previous cystinosis mouse model studies that detected pronounced accumulation of cystine crystals in the choroid and INL using transmission electron microscopy scans. ${ }^{20}$ The INL and GCL contain cell nuclei and perikarya of ganglion cells, amacrine cells, bipolar cells and horizontal cells. ${ }^{21}$ Since it is assumed that cystine crystals are primarily deposited in the intracellular compartment, ${ }^{22}$ the pronounced deposition in the GCL and INL may be related to the relative abundance of cell nuclei in these layers. Interestingly, crystals within the INL were predominantly found at the transition zones with the adjacent IPL and OPL, both representing regions with an abundance of retinal microvasculature. The vasculature of the retina is divided into three microvascular plexus: superficial, intermediate and deep. These are localised within the RNFL and in the IPL/INL and INL/OPL transition zones, respectively. Distinct crystal accumulation within pericytes has been demonstrated with electron microscopy of brain tissue of a patient with cystinosis. ${ }^{23}$ Pericytes coat the microvascular endothelial cells and their functional role as macrophages has been recognised. ${ }^{24}$ Moreover, it has been shown that pericytes contain a notable number of lysosomes-a feature that characterises the ultrastructure of macrophages. ${ }^{25}$ Hence, we hypothesise that the pronounced crystal accumulation in the GCL and INL may be attributable to cystine-loaded pericytes in the respective retinal capillary plexus. Of note, as compared with the GCL and INL, even more abundant crystal accumulation was detected in the highly vascularised choriocapillaris. In analogy to the neuroretina, crystals appeared to deposit primarily in a perivascular distribution in the choriocapillaris layer (figure 2). The appearance of perivascular region may go hand in hand with localisation in pericytes, although a previous histological study also localised crystal accumulation to the choroidal endothelial cells. ${ }^{26}$ SD-OCT can only confirm the crystals' perivascular localisation and hypothesise on their exact cellular origin. Histological examinations are needed to precisely differentiate the perivascular cell types, that is, choroidal endothelial cells, pericytes or perivascular innate immune cells in terms of resident choroidal macrophages. ${ }^{27}$
Since cystine crystal accumulation in corneal tissue is known to correlate poorly with visual function, retinal crystal accumulation has been held responsible for the loss of vision in patients with cystinosis. $^{2829}$ The findings of the present study support this hypothesis. RCCCS was negatively associated with visual acuity, and positively associated with the severity of patients' glare perception (figures 4 and 5). Of note, cystinosis retinopathy may not only account for visual acuity loss, but contribute to photophobia, which is considered to be one of the main visual burdens of patients with cystinosis. ${ }^{5}$

Adherence to systemic treatment is crucial, because it can prevent or delay end organ damage in almost every tissue, including the retina. ${ }^{30}$ Therefore, particular efforts need to be undertaken to foster compliance with systemic therapy, which may be encouraged by precise monitoring of disease control as a feedback mechanism for patients. Corneal cystine crystals can only be dissolved with topical cystine-depleting therapy and, hence, are not suitable as biomarkers for systemic disease control. Tsilou et al were the first to acknowledge the retina as a 'window' to the systemic manifestations of cystinosis. ${ }^{28}$ The authors reported a direct correlation between the incidence of cystinotic retinopathy, as determined by funduscopy, perimetry or electroretinography, and the time period that the patients did not receive oral cysteamine therapy. In their study, however, traditional funduscopy was used to screen for retinal crystals. In our study using high-resolution SD-OCT, retinochoroidal crystals could be seen in all examined eyes, including those of asymptomatic paediatric patients. With respect to the predominant deposition locus of crystals in the choriocapillaris underlying the RPE, the crystal burden may be overestimated with conventional funduscopy. A low degree of crystal accumulation may not necessarily lead to functional defects and, thus, may not be picked up by ERG or perimetry in the early stages. In addition, perimetry and ERG are generally not feasible in patients with photophobic paediatric cystinosis due to the lengthy acquisition time and the bright flashlight stimuli, respectively. ${ }^{31}$

SD-OCT represents an objective, fast and contact-less imaging method operating in the infrared spectrum, which is invisible for the human eye and does not elicit photophobia. Based on the aforementioned findings, we conclude that the SD-OCT based RCCCS system may accurately reflect the extent of retinochoroidal cystinosis disease even at an early stage. Moreover, a plurality of findings from the present study indicates that the RCCCS system may also serve as a valuable biomarker for systemic disease status and control. First, RCCCS showed a statistically significant association with cystatin C (figure 4A), a stable endogenous protein used to determine renal function that is less dependent on the patient's age and muscle mass as compared with the commonly used creatinine parameter. Second, RCCCS was negatively influenced by the daily cysteamine dose. Eyes of patients with a known low therapy compliance (marked in red) or of patients with cysteamine intolerance (marked in blue), showed particularly high RCCCS values (figure 4B). Moreover, the RCCCS tended to increase with higher WBC cystine levels, currently the sole biomarker to monitor oral cystinosis therapy (figure 4C). Third, as cystinosisrelated end organ damage generally progresses with age, older patients showed a propensity towards higher RCCCS values. Finally, the most pronounced cystine crystal accumulation was found in patients harbouring the $57-\mathrm{kb}$ deletion. This is the most common mutation found in patients of European descent. It is also referred to as a 'severe nephropathic type' of mutation and causes the most severe cystinotic nephropathy. ${ }^{32}$ Hence, although not directly assessed in this study, the RCCCS may 
be an early parameter indicating advancing nephropathic and systemic damage.

Of interest, in our patient collective, the RCCCS did not correlate with the subjective Gahl CCCS nor with the objective grey scale based grading system developed by our group.

This is most likely because the cornea is not connected to the systemic circulation and thus corneal cystine crystal deposition is not affected by oral therapy. For this reason, corneal cystine crystals are treated with eye drops containing cysteamine. In our experience, treatment compliance for taking the cysteaminecontaining tablets to treat the systemic manifestations differs significantly from the compliance for eye drop application. Many patients are negligent in the use of the eye drops, but still take the vital tablets regularly. Accordingly, a high number of chorioretinal crystals does not necessarily entail an increased occurrence of corneal cystine crystals.

One limitation of this study is that the RCCCS system represents a semiquantitative scoring model that requires an expert SD-OCT reader. With the help of deep learning based retinal OCT segmentation, however, objective and fully automated scoring of retinochoroidal cystine crystals may be possible in the near future. ${ }^{33}$ Similarly, we could not take OCT scans in very young patients due to lack of compliance. In future studies, this could be accomplished with the help of handheld OCT systems. ${ }^{34}$ A strength of this study is its relatively large sample of 85 eyes. Infantile nephropathic cystinosis has an estimated incidence of 1:100 000-1:200 000. ${ }^{12}$ In all of Germany, approximately 130 patients are currently known and 3-5 new patients are diagnosed per year. ${ }^{35}$ Therefore, the presented cohort of patients with cystinosis may be regarded as highly valuable in broadening our understanding of this rare genetic disorder.

In conclusion, the herein introduced RCCCS system may accurately reflect the extent of retinochoroidal cystinosis disease at every stage of the disease. Moreover, our findings indicate that the RCCCS system could serve as a valuable biomarker for systemic disease status and cysteamine therapy adherence. Longitudinal clinical studies incorporating the novel RCCCS system are warranted to explore the temporal dynamics between retinochoroidal crystal deposition and systemic disease control.

Collaborators German Cystinosis Study Group: Bechtold Dalla Pozza S, Bergmann C, Buss M, Dosch R, Erler J, Getzinger T, Herzig N, Holla H, Knerr C, Koeppl C, Ockert C, Passow M, Rohayem J, Steidle G, Thiele A, Treikauskas U, Weber R, Weitzel D.

Contributors All authors conceived and planned the study. LK, CP and BS performed the ophthalmological examinations. LK and CP analysed the data. All authors contributed to the interpretation of the results. LK, CP and NL wrote the manuscript with input from all authors. All authors provided critical feedback and helped shape the research, analysis and manuscript.

Funding The authors have not declared a specific grant for this research from any funding agency in the public, commercial or not-for-profit sectors.

Competing interests LK received previous speaker fees and/or travel expenses from Recordati Rare Diseases and Roche Diagnostics GmbH. KH received previous speaker fees from Recordati Rare Diseases. BS received previous speaker fees and travel expenses from Novartis Pharma GmbH and Topcon Corporation. SP received previous speaker fees and/or travel expenses from Novartis Pharma GmbH, Oertli AG, Bayer AG, Alcon Pharma GmbH and Pharm-Allergan GmbH. NL received income from honoraria as a lecturer from Alcon Laboratories, NIDEK Co., and CenterVue SpA. CP received previous speaker fees from Novartis Pharma GmbH and travel expenses from Recordati Rare Diseases Inc.

\section{Patient consent for publication Obtained.}

Ethics approval All research and measurements followed the tenets of the Declaration of Helsinki. Ethics committee approval for this study was waived by the ethics committee (Ethikkommission der Bayerischen Landesärztekammer; 11 March 2015) because the planned project of an interdisciplinary cystinosis database does not fall under the consultation obligation according to paragraph 15 of the professional code of conduct for physicians in Bavaria, Germany.
Provenance and peer review Not commissioned; externally peer reviewed.

Data availability statement Data are available in a public, open access repository. Repository name: The Open Science framework URL: https://osf.io/ 3xbmd/?view_0

Open access This is an open access article distributed in accordance with the Creative Commons Attribution Non Commercial (CC BY-NC 4.0) license, which permits others to distribute, remix, adapt, build upon this work non-commercially, and license their derivative works on different terms, provided the original work is properly cited, appropriate credit is given, any changes made indicated, and the use is non-commercial. See: http://creativecommons.org/licenses/by-nc/4.0/.

\section{ORCID iD}

Leonie Keidel http://orcid.org/0000-0003-4144-5754

\section{REFERENCES}

1 David D, Princiero Berlingerio S, Elmonem MA, et al. Molecular basis of cystinosis: geographic distribution, functional consequences of mutations in the CTNS gene, and potential for repair. Nephron 2019:141:133-46.

2 Wilmer MJ, Emma F, Levtchenko EN. The pathogenesis of cystinosis: mechanisms beyond cystine accumulation. Am J Physiol Renal Physiol 2010;299:F905-16.

3 Foreman JW. Fanconi syndrome. Pediatr Clin North Am 2019;66:159-67.

4 Cherqui S, Courtoy PJ. The renal fanconi syndrome in cystinosis: pathogenic insights and therapeutic perspectives. Nat Rev Nephrol 2017;13:115-31.

5 Gahl WA, Kuehl EM, Iwata F, et al. Corneal crystals in nephropathic cystinosis: natural history and treatment with cysteamine eyedrops. Mol Genet Metab 2000;71:100-20.

6 Keidel L, Elhardt C, Hohenfellner K, et al. Establishing an objective biomarker for corneal cystinosis using a threshold-based spectral domain optical coherence tomography imaging algorithm. Acta Ophthalmol 2021;99:e189-95.

7 Dureau P, Broyer M, Dufier J-L. Evolution of ocular manifestations in nephropathic cystinosis: a long-term study of a population treated with cysteamine. J Pediatr Ophthalmol Strabismus 2003;40:142-6.

8 Thoene JG, Oshima RG, Crawhall JC, et al. Cystinosis. intracellular cystine depletion by aminothiols in vitro and in vivo. J Clin Invest 1976;58:180-9.

9 Kimonis VE, Troendle J, Rose SR, et al. Effects of early cysteamine therapy on thyroid function and growth in nephropathic cystinosis. J Clin Endocrinol Metab 1995;80:3257-61.

10 Kleta R, Gahl WA. Pharmacological treatment of nephropathic cystinosis with cysteamine. Expert Opin Pharmacother 2004;5:2255-62.

11 Nesterova G, Gahl W. Nephropathic cystinosis: late complications of a multisystemic disease. Pediatr Nephrol 2008;23:863-78.

12 Elmonem MA, Veys KR, Soliman NA, et al. Cystinosis: a review. Orphanet J Rare Dis 2016;11:47.

13 Biswas S, Sornalingam K. The ocular status of cystinosis patients receiving a hospital pharmacy-made preparation of cysteamine eye drops: a case series. Ophthalmol Ther 2019;8:125-36.

14 Fujimoto JG, Pitris C, Boppart SA, et al. Optical coherence tomography: an emerging technology for biomedical imaging and optical biopsy. Neoplasia 2000;2:9-25.

15 Kaiser-Kupfer MI, Caruso RC, Minkler DS, et al. Long-term ocular manifestations in nephropathic cystinosis. Arch Ophthalmol 1986;104:706-11.

16 Kozak I, Arevalo JF, Shoughy SS. Intraretinal crystals in nephopathic cystinosis and fanconi syndrome. JAMA Ophthalmol 2017;135:e165169.

17 Hohenfellner K, Deerberg-Wittram J, Coordinated D-WJ. Coordinated, cost-effective care for rare disease: the cystinosis outpatient consultation program at RoMed. NEJM Catalyst 2020;1.

18 McAlinden C, Pesudovs K, Moore JE. The development of an instrument to measure quality of vision: the quality of vision (QoV) questionnaire. Invest Ophthalmol Vis Sci 2010;51:5537-45

19 Schneider JA, Verroust FM, Kroll WA, et al. Prenatal diagnosis of cystinosis. N Engl J Med 1974:290:878-82.

20 Kalatzis V, Serratrice N, Hippert C, et al. The ocular anomalies in a cystinosis animal model mimic disease pathogenesis. Pediatr Res 2007;62:156-62.

21 Gupta MP, Herzlich AA, Sauer T, et al. Retinal anatomy and pathology. Dev Ophthalmol 2016:55:7-17.

22 Iwata F, Kuehl EM, Reed GF, et al. A randomized clinical trial of topical cysteamine disulfide (cystamine) versus free thiol (cysteamine) in the treatment of corneal cystine crystals in cystinosis. Mol Genet Metab 1998;64:237-42.

23 Vogel DG, Malekzadeh MH, Cornford ME, et al. Central nervous system involvement in nephropathic cystinosis. J Neuropathol Exp Neurol 1990;49:591-9.

24 Thomas WE. Brain macrophages: on the role of pericytes and perivascular cells. Brain Res Brain Res Rev 1999;31:42-57.

25 Farrell CR, Stewart PA, Farrell CL, et al. Pericytes in human cerebral microvasculature. Anat Rec 1987:218:466-9.

26 Tsilou E, Zhou M, Gahl W, et al. Ophthalmic manifestations and histopathology of infantile nephropathic cystinosis: report of a case and review of the literature. Surv Ophthalmol 2007;52:97-105. 
27 Forrester JV, Xu H, Kuffová L, et al. Dendritic cell physiology and function in the eye. Immunol Rev 2010;234:282-304.

28 Tsilou ET, Rubin BI, Reed G, et al. Nephropathic cystinosis: posterior segment manifestations and effects of cysteamine therapy. Ophthalmology 2006;113:1002-9.

29 Dufier JL, Dhermy P, Gubler MC, et al. Ocular changes in long-term evolution of infantile cystinosis. Ophthalmic Paediatr Genet 1987:8:131-7.

30 Brodin-Sartorius A, Tête M-J, Niaudet P, et al. Cysteamine therapy delays the progression of nephropathic cystinosis in late adolescents and adults. Kidney Int 2012:81:179-89.

31 Young B, Eggenberger E, Kaufman D. Current electrophysiology in ophthalmology: a review. Curr Opin Ophthalmol 2012;23:497-505.
32 Anikster Y, Shotelersuk V, Gahl WA. Ctns mutations in patients with cystinosis. Hum Mutat 1999:14:454-8.

33 Pekala M, Joshi N, Liu TYA, et al. Deep learning based retinal OCT segmentation. Comput Biol Med 2019:114:103445.

34 Scott AW, Farsiu S, Enyedi LB, et al. Imaging the infant retina with a handheld spectral-domain optical coherence tomography device. Am J Ophthalmol 2009;147:364-73.

35 Hohenfellner K, Bergmann C, Fleige T, et al. Molecular based newborn screening in Germany: follow-up for cystinosis. Mol Genet Metab Rep 2019;21:100514.

36 Hunter JJ, Morgan JIW, Merigan WH, et al. The susceptibility of the retina to photochemical damage from visible light. Prog Retin Eye Res 2012;31:28-42. 